\title{
Décollectivisation et relations de propriété dans un village roumain
}

Land Restitution and Property Relations in a Romanian village

\section{Florina Gaborean}

\section{(2) OpenEdition}

Journals

\section{Édition électronique}

URL : http://journals.openedition.org/economierurale/3280

DOI : $10.4000 /$ economierurale.3280

ISSN : 2105-2581

\section{Éditeur}

Société Française d'Économie Rurale (SFER)

\section{Édition imprimée}

Date de publication : 21 octobre 2011

Pagination : 114-128

ISSN : 0013-0559

\section{Référence électronique}

Florina Gaborean, « Décollectivisation et relations de propriété dans un village roumain », Économie rurale [En ligne], 325-326 | septembre-décembre 2011, mis en ligne le 17 octobre 2013, consulté le 01 mai 2019. URL : http://journals.openedition.org/economierurale/3280 ; DOI : 10.4000/

economierurale.3280 


\section{Décollectivisation et relations de propriété dans un village roumain}

Florina GABOREAN • Institut national de la recherche scientifique, Centre-Urbanisation, Culture et Société, Montréal (Québec), Canada

florina_gaborean@ucs.inrs.ca

\section{Introduction}

a restitution du droit de propriété privée a constitué une priorité des gouvernements post-socialistes dans les années 1990. Le monde rural a été parmi les secteurs sociaux les plus touchés par ces politiques de privatisation. Les réformes engagées dans ce secteur sont désignées dans la littérature scientifique sous le nom de décollectivisation, vue comme une mutation des droits de propriété et des rapports sociaux affectant à l'intérieur du système tous les facteurs de production (terre, capital, travail) qui interviennent dans le fonctionnement des exploitations agricoles (Maurel, 1994). Ce processus renvoie à une idée de retour à l'avant-collectivisation, à l'ancien ordre social fondé sur l'appropriation privée du sol et des moyens de le cultiver. Dans l'ensemble des pays de l'Europe de l'Est, la restauration de la propriété privée de la terre a constitué un élément central de la transition post-socialiste.

Cette importance accordée à la propriété privée comme moteur de développement social et économique a été intégrée par une bonne partie de la littérature scientifique consacrée aux pays post-socialistes. La transition est alors conçue comme un passage d'un modèle social et économique socialiste vers un modèle économique et social libéral : transition vers la démocratie et l'économie de marché. L'analyse d'un changement social complexe a été réduite ou subordonnée aux postulats simplificateurs d'un changement politique et économique dont la nature et les orientations semblaient connues à l'avance (Frybes, 1998).
Vingt ans après la chute du communisme, on constate que cette vision unilinéaire de la transition est confrontée à de nombreux défis. Premièrement, la mise en place des structures agricoles performantes basées sur la propriété privée a été plus longue et difficile que prévue. On constate l'apparition d'économies hybrides, fluides et plurielles qui ne sont plus collectives, ni encore complètement intégrées au marché (Gambold, 2008).

Deuxièmement, les critiques du néolibéralisme ont remis en question cette interprétation de la transition. En contexte postsocialiste, les relations de propriété ne suivent pas une voie unique et prédictible vers le capitalisme mais témoignent des variations significatives dues à l'héritage social et culturel de chaque pays. Une juste compréhension de ces transformations devrait prendre en compte les choix des acteurs, leurs stratégies et leurs pratiques dans la construction de ces nouvelles réalités sociales (Hann, 2002 ; Verdery, 2003).

Troisièmement, les analyses anthropologiques fondées sur des recherches empiriques constatent que les relations de propriété varient grâce à leur enchâssement dans des relations sociales. La littérature anthropologique considère que la propriété donne forme et substance aux relations entre les gens en ce qui touche les objets de valeurs. Trois éléments sont constitutifs de ces relations : les unités sociales (individus, sociétés, États), les valeurs conçues comme objets de propriété et enfin, les droits et obligations rattachées à ces objets. Empiriquement, « la propriété trouve son expression dans des couches d'organisation sociales, 
c'est-à-dire que les pratiques sociales créent, maintiennent et transforment ce qui est la propriété au niveau des idéologies, des règles institutionnalisées en droit et des relations réelles. Au sein d'un régime de propriété, chacun des niveaux (idéologies, réglementations, relations) peut changer avec des vitesses différentes et pour des raisons différentes (Turner, Wiber, 2009). Pour les acteurs ruraux, la contrainte qu'ils subissent réellement des idéologies, des institutions légales et des liens de propriété varie à cause des facteurs historiques, politiques, économiques et sociaux. Ainsi, les traditions juridiques locales (règles de transmission et de succession), le rapport à la terre et l'usage du paysage rural peuvent influencer les transformations de la propriété rurale.

Cet article s'inscrit dans cette perspective anthropologique qui met en évidence l'enchâssement social des relations de propriété. Il cherche à jeter un nouvel éclairage sur les changements produits dans les campagnes roumaines depuis la décollectivisation, processus qui a entrâné une véritable réforme agraire et une profonde transformation du monde rural. Fondé sur plusieurs années de recherche de terrain, cet article examine les modifications des relations de propriété dans un village de Transylvanie pendant 20 ans de transition postcommuniste.

Notre regard anthropologique va porter une attention particulière à la signification sociale et culturelle de la propriété rurale et à la manière dont les acteurs ruraux incorporent les changements sociaux au niveau des relations de propriété. Dans un premier temps, nous présenterons les règles d'accès à la propriété privée après la chute du communisme ainsi que le cadre juridique. Ces éléments serviront de point de départ à la compréhension de la spécificité de la décollectivisation en Roumanie et des transformations des relations de propriété en contexte post-socialiste. Dans un deuxième temps, nous analyserons les principales données empiriques issues de notre enquête ethnographique. Nous montrerons que la résurgence des coutumes, dans les représentations et les pratiques des acteurs ruraux (transmission patrimoniale, usages de la terre), a joué un rôle central dans la recomposition des rapports de propriété au niveau local. Les transformations post-socialistes de la propriété rurale suivent des principes et des pratiques héritées à la fois du passé socialiste et du passé pré-collectiviste.

\section{La législation roumaine post-collectiviste}

Pays d'économie encore très agraire ${ }^{1}$, la Roumanie a subi de plein fouet les politiques de privatisation après la chute du communisme, car $90 \%$ de la terre a été collectivisée dans les années $1950^{2}$. La collectivisation a entraîné des transformations sociales importantes dans tous les secteurs sociaux : l'exode rural, l'accroissement démographique urbain, les migrations journalières et hebdomadaires entre ville et campagne. Elle a remodelé l'espace géographique par l'introduction d'une agriculture mécanisée et de grande surface, et a engendré un mode particulier d'articulation sociale entre ville et village.

La dynamique de ce processus s'est réalisée dans un cadre social et géographique spécifique. Les Roumains disposent de deux unités importantes d'organisation sociale : la gospodaria et le village. La gospodaria comprend le groupe domes-

1. Selon une enquête réalisée en 2008 par le ministère de l'Agriculture et du Développement rural, 45,6\% de la population habite encore à la campagne. La population active pour $27,7 \%$ travaille en agriculture, ce qui permet à près de 4 millions de ménages de vivre grâce à une agriculture de subsistance. 2. En Roumanie, la collectivisation est un processus profond et de longue durée. Dans la période allant de 1945 à 1962, 10 millions d'hectares de terrains agricoles sont devenus propriété des coopératives socialistes. La Roumanie est aussi le second pays après l'URSS où la politique de systématisation des villages s'est plus développée, traduisant un durcissement du régime communiste (Civici, Jouve, 2009). 
tique, la cour avec la maison, les dépendances et un lopin jardiné. Elle représente l'élément essentiel autour duquel s'organisent toutes les activités du monde rural roumain. Le village inclut plusieurs éléments : un groupe de personnes installées dans un seul habitat disposant de propriétés intravilan (situées au noyau du finage villageois et qui appartient à la gospodaria) et d'un territoire extravilan (situé à l'extérieur du finage villageois constitué de terres arables, prairies et pâturages). Pendant le communisme, les terres extravilan ont été collectivisées alors qu'une partie des terres intravilan est restée dans le patrimoine familial. Après la chute du communisme, les terres extravilan et une partie des terres intravilan ont été restituées aux anciens propriétaires.

Dans ce contexte, au début des années 90, la décollectivisation a constitué un enjeu national de premier ordre. Avec un effectif de plus de 10 millions de personnes, les populations rurales ont été les premières à subir les effets de la transition caractérisés par un bouleversement rapide des structures foncières (Hirschhausen, 1997).

Visant à mettre fin aux aberrations du système coopératif et à faire sortir le pays des pénuries alimentaires, le Front du salut national $^{3}$ a promulgué le décret $42 \mathrm{du}$ 29 janvier 1990. Sans toucher au principe de la propriété collectiviste des terres, il a permis aux paysans des coopératives d'agrandir leurs lopins individuels, réduits sous le régime communiste, en attribuant l'usufruit de 50 ares à chaque membre des coopératives et 25 ares aux salariés des fermes d'État et aux employés dans les zones rurales.

Dans ses analyses sur le processus de décollectivisation en Transylvanie, Hirschhausen (op. cit.) observe que ces mesures législatives prudentes ont été largement dépassées au plan local par des mouvements spon-

3. Le parti qui a pris le pouvoir après la chute du régime communiste. tanés de dissolution des coopératives, engagés sur l'initiative des villageois. Ces mouvements « révolutionnaires » ont remis en cause les fondements même des principes collectivistes. Ils ont été accompagnés par la brusque réorganisation du travail au sein des coopératives, ce qui a conduit à l'apparition de grandes surfaces laissées en friche et à une crise de l'organisation coopérative. À la suite de ce décret, l'agriculture familiale a fortement progressé par le développement du marché paysan et par la multiplication des échanges directs entre la ville et la campagne. En contrepartie, le décret a gravement marginalisé l'appareil étatique de collecte et de distribution agroalimentaire qui auparavant détenait le monopole socialiste dans ce domaine (1997).

La mise en application de cette mesure a entraîné dès la fin de l'année 1990, un accroissement de 2,2 millions d'hectares de la surface agricole privée. En fait, le décret 42 a abouti, notamment dans la région de Transylvanie où les paysans possédaient encore des cadastres, à une privatisation spontanée des terres (Lhomel, 1995). On peut donc le considérer comme précurseur de la loi 18, dite Legea fondului funciar (loi du fonds foncier).

La loi 18/1991 prévoit la restitution des propriétés aux anciens propriétaires dépossédés par la collectivisation et en cas de décès, à leurs héritiers. Le texte de la loi fait référence à la situation foncière du début des années 50, d'autant plus qu'elle autorise la restitution des terres sur les anciens emplacements. Elle fixe la limite de 10 hectares de terrain agricole par famille et d'un hectare de forêt, indépendamment de la surface que la famille avait détenue avant la collectivisation. La loi accorde également une parcelle de 0,5 à 1 ha aux membres des coopératives agricoles de production qui n'en possédaient pas avant la collectivisation, à condition d'avoir un domicile permanent dans le village et de mettre la superficie reçue en culture. Les mécaniciens et les cadres intermédiaires de la coopérative bénéficient du 
même droit (article 13). Lorsque la quantité de terres n'est pas suffisante, un coefficient de réduction est affecté aux autres ayants droit (article 19). La loi a ainsi valorisé une justice réparatrice et une justice distributive ${ }^{4}$. Pour parer à un possible « émiettement » du paysage agricole, la loi prévoit également la constitution d'associations agricoles privées mais sans préciser le cadre légal. La loi sur les sociétés agricoles et autres formes d'associations en agriculture a été promulguée deux mois plus tard ${ }^{5}$. En janvier 2000, la loi 1 a modifié la limite supérieure de restitution des terres prévue par la loi 18/1991. On a restitué aux ayants droit jusqu'à 50 hectares de terrain agricole et 10 hectares de forêt. La loi a également prévu la liquidation des anciennes entreprises d'État transformées en sociétés commerciales.

Ces mesures législatives n'ont pas donné les résultats escomptés. En l'absence d'un appui économique et technique de la part de l'État, les paysans ont recommencé à travailler les terres en recourant à des méthodes et à des savoirs traditionnels. La fragmentation des exploitations, le manque de moyens et le recours aux instruments de travail ancestraux marquent ce que les analystes ont appelé « le retour paysan ».

4. Une analyse des lois qui ont mené à la privatisation des terres dans les pays post-socialistes révèle l'affrontement entre deux conceptions de la justice sociale. L'une s'appuie sur la notion de réparation morale et matérielle pour demander la restitution des biens à leurs anciens propriétaires. C'est la conception de la « justice réparatrice ». L'autre a proposé d'attribuer des parcelles aux travailleurs sans terre sur le critère de l'apport en travail. C'est une forme de justice «distributive » (Maurel, op.cit.).

5. Cette loi rend possible plusieurs formes d'associations : sociétés commerciales qui prévoient la transformation des ex-fermes d'État (Intreprinderi Agricole de Stat - IAS) en sociétés par actions à $100 \%$ capitaux d'État ; associations familiales conclues à la base d'un accord signé par plusieurs familles et associations dites « sociétés agricoles », entités juridiques reconnues constituées par un nombre variable et non limité d'associés (dix au minimum).
Des études réalisées sur le terrain [Amblard, Colin (2006) ; Stan (2005) ; Cartwright (2001) ; Lhomel (op. cit.) ; Mihailescu (2000 ; 1996) ; Sandu (1999)] montrent également qu'à l'exception des zones non-collectivisées, où prédominent les exploitations de type familial dans l'ensemble des autres régions, coexistent dans une même localité (communes, villages) plusieurs types d'exploitations (associations familiales informelles ou juridiques, agriculteurs indépendants, sociétés agricoles constituées d'entrepreneurs privés qui ont pris les terres en bail). L'intégration dans l'Union européenne n'a pas beaucoup changé les données. Malgré la mise en place de certains programmes de développement rural, l'agriculture de subsistance et le travail familial continuent de représenter les principales sources de revenus pour la population active en milieu agricole.

Ces résultats peu convaincants de la réforme agricole dans les campagnes roumaines sont analysés par plusieurs chercheurs autochtones et occidentaux. Différentes explications ont été avancées :

- la discordance entre les enjeux nationaux et locaux de la décollectivisation (Hirschhausen 1997, 1999) ;

- la politique étatique populiste et inefficace (Otiman, 1994) ;

- le manque d'un modèle agricole (Mihailescu, 1996) ;

- la concentration du pouvoir au niveau des autorités locales ;

- la corruption des maires et des commissions locales de privatisation et l'héritage collectiviste (Verdery, 1997, 2002a, 2002b).

À la limite, la réforme est vue comme « un facteur de déconstruction et d'effondrement du monde rural » (Badescu, 2003). Ces analyses se concentrent plutôt sur la mise en application de la réforme agraire et sur les comportements économiques des acteurs sociaux, tout en minimisant le rôle joué par le groupe domestique, et les 
pratiques de succession et d'héritage. Cependant, la législation post-collectiviste a placé l'héritage de la propriété foncière au centre de la réforme agricole. Les lois $18 / 1991$ et $1 / 2000$ ont pris en compte les structures foncières des années 50 . Or, la plupart des sujets juridiques de ces lois, les anciens propriétaires, étaient décédés, ce qui a transféré les responsabilités vers les héritiers légitimes. De cette façon, l'application de la loi a créé dans la pratique un double effet juridique : la rétrocession et la transmission des terres ont été gérées simultanément (Gaborean, 2008). Dans ce contexte, l'héritage et les relations de parenté ont pris une importance nouvelle en contexte post-socialiste : la société roumaine a été, dans sa quasi-totalité, concernée par la restitution des terres.

\section{Lieu de la recherche et méthodologie}

Notre approche est qualitative et la méthode utilisée est empirique. Elle est fondée sur des investigations profondes, de longue durée, dont les résultats n'illustrent pas nécessairement tous les cas qui peuvent exister en réalité. Ainsi, « la représentativité dont il s'agit ici ne résulte pas de formes de généralisation statistique ou prédictive. Elle est qualitative et inductive, visant à expliquer des mécanismes ainsi que des processus » (Le Meur, 2002).

Nos données ethnographiques ont été recueillies dans le village de Cerghid ${ }^{6}$, une communauté paysanne de 486 habitants située au centre-nord de la Transylvanie, à proximité de deux centres urbains de première importance pour cette région:

6. Les données empiriques, dont fait l'objet cet article, relèvent de nos recherches doctorales. La recherche principale sur le terrain a été réalisée à plusieurs reprises entre 2001 et 2005 mais le contact que nous avons gardé avec cette communauté nous a permis de suivre les changements tout au long de la période post-socialiste.
Târgu-Mures et Tirnaveni. Le village en question a été entièrement collectivisé et a connu un important exode rural pendant le communisme 7 . Cette communauté peut être représentative du rapport entre milieu urbain et rural par le biais des maisonnées et des parentèles qui ont gardé un ancrage dans le milieu rural et pour l'étude d'une catégorie sociale hybride apparue après 1989 qui est constituée des propriétaires qui habitent la ville mais qui travaillent les terres restituées à la campagne.

Les méthodes d'investigation ont combiné les entretiens semi-directifs et non directifs, l'étude des archives familiales et départementales (documents d'état civil, registres fonciers et cadastres, testaments, titres de propriété) et la reconstitution des généalogies portant sur trois générations. Des entretiens répétés ont été menés auprès de 25 couples mariés (50 personnes) sélectionnés en fonction de trois variables (la catégorie de maisonnées, la catégorie de propriétaires fonciers et le cycle de développement du groupe domestique) croisées avec l'usage et l'occupation de la terre. Nous avons également réalisé des entretiens avec les autorités locales et les membres de la mairie impliqués dans le processus de restitution des terres (5 personnes concernées). Nous avons réalisé une analyse comparée des registres agricoles et des cadastres avant, pendant et après la collectivisation. L'étude de ces sources a contribué à une meilleure compréhension des changements opérés dans la structure de la propriété foncière.

7. La population passe de 809 habitants en 1949 à 600 habitants en 1966. Les villes industrialisées situées à proximité du village ont absorbé une partie importante des jeunes actifs. Plusieurs personnes ont choisi d'habiter le village mais de travailler en ville. Pendant le communisme, l'agriculture est devenue une affaire de retraités, de femmes ou bien une activité complémentaire à la fin des journées à l'usine. 


\section{Les pratiques de transmission au cœur de la restitution des propriétés}

Plusieurs chercheurs qui ont analysé la propriété rurale ont constaté que la transmission des biens constitue l'un des principaux éléments d'un vaste processus, à travers lequel, se reproduisent les rapports de propriété. Derouet et Goy (1998) ont montré que dans les sociétés rurales, la terre ne peut pas être réduite à son seul aspect économique, de bien de production. Elle doit être envisagée aussi pour son rôle éventuel de capital symbolique. Ainsi, la terre peut être objet de plusieurs traitements, pratiques et représentations. La terre n'est pas en soi un bien « immeuble » mais elle le devient par l'usage social qui lui est réservé, parce qu'on fait (Dérouet, Goy, op. cit.). Les trois sections qui suivent mettent en relation les pratiques de transmission patrimoniale, les usages sociaux des terres et les représentations associées à la propriété rurale dans le village de Cerghid. Ces éléments expliquent l'enchâssement social de la propriété foncière en contexte postsocialiste.

Après la chute du communisme, dans le village de Cerghid, la restitution des terres aux anciens propriétaires a constitué l'un des problèmes les plus importants de la communauté. La plupart des demandes de restitution (273) relevaient de la reconstitution du droit à la propriété pour une surface de 810 ha de terrain arable. De ce nombre de demandes, 110 ont été faites par des héritiers des anciens propriétaires résidant actuellement à l'extérieur du village. Cela donne un bon aperçu de l'ampleur de ce phénomène de restitution qui a bien dépassé le cadre restreint du village. En vertu de l'article 13 de la loi 18/1991, 22 personnes (10 villageois, 12 techniciens et spécialistes de la coopérative) ont reçu des terres. La mise en application de cette disposition a entraîné une réduction de $3 \%$ des terres restituées à l'ensemble du village, ce qui a provoqué le mécontentement des propriétaires légitimes.

Cette redistribution a placé l'héritage de la propriété foncière au centre des préoccupations des membres de la gospodaria. Les gens sont devenus conscients de l'enjeu de l'héritage. Beaucoup de propriétaires qui habitaient en ville sont revenus au village pour travailler les terres, ce qui a renforcé les liens entre les membres de la gospodaria. Les activités d'entraide se sont multipliées :

"Quelques années après la révolution, le champ était plein de citadins qui se sont procurés des bêches et qui travaillaient une partie des terres restituées. Dans ce temps-là, les membres de la parenté, même ceux qui sont partis en ville, venaient chaque automne pour partager les céréales et tout le monde avait de grands espoirs. » (Vasile Merca ${ }^{8}, 61$ ans)

En sus des solidarités familiales, ce processus a donné naissance à plusieurs conflits et tensions entre héritiers. Plus de la moitié des familles interviewées ont évoqué des conflits avec un neveu lointain, avec une tante oubliée ou avec un frère proche. L'analyse de ces situations montre que les litiges sont provoqués par le refus des villageois de donner la terre de bonne qualité à ceux qui habitent en ville ou par la tendance à la monopolisation de l'un des héritiers. Ici, les conflits mettent en jeu les différences de perception et d'interprétation entre le droit civil égalitaire et les pratiques coutumières inégalitaires :

«Je pense que la loi qui prévoit un partage égal pour n'importe quelle situation n'est pas juste. Les gens partis en ville pendant le communisme ont oublié le travail dur de la terre. Il faut que les frères s'entendent entre eux et reconnaissent les mérites de ceux qui sont restés au village et qui ont enduré toutes les difficultés $d u$ collectivisme. » (Anca Rarau, 73 ans)

Aux yeux des villageois, les pratiques coutumières inégalitaires ont plus de légi-

8. Afin de protéger l'anonymat des personnes interviewées, les noms attribués sont fictifs. 
timité que les dispositions juridiques. Alexandra Gal (66 ans) fait une nette confusion entre les règles instituées par le droit civil et celles que dicte la coutume. Selon elle, la loi, c'est la coutume :

«Je ne connais pas la loi maintenant, mais je sais qu'avant la collectivisation il y avait la loi suivante: l'enfant qui prenait soin de ses parents jusqu'à leur mort héritait de la maison et d'une grande partie des terres. De nos jours, les frères demandent des parties égales même s'ils n'ont pas pris soin de leurs parents. Cette loi n'est pas juste, elle doit reconnaître les mérites de l'enfant qui est resté auprès des parents. »

Mais cette confusion, entre loi et coutume, entre en conflit avec la vision de certains héritiers installés en ville qui veulent tout simplement obtenir le droit de propriété conféré par la loi. Cette différence de perception entre les héritiers citadins et ceux qui sont restés au village à l'égard de la légitimité de l'accès à la terre est un aspect qui a considérablement ralenti la redistribution foncière. En restituant le titre de propriété aux anciens propriétaires ou à l'un des héritiers, la loi a favorisé le retour à l'indivision foncière, une pratique coutumière existante avant la collectivisation. Prenons l'exemple du villageois Gheorghe Catalin qui a trois sœurs et un frère, tous partis en ville pendant le socialisme. C'est lui qui fait la demande de restitution. Son nom figure sur le titre de propriété avec la spécification, au verso, qu'il y a encore quatre héritiers qui ont le même droit. La surface restituée est de 8 ha 19 ares morcelés en 8 parcelles. ${ }^{9}$ Selon la

\footnotetext{
9. Avant la collectivisation, les propriétés arables en extravilan étaient excessivement morcelées. La taille d'une propriété moyenne était de 3-4 hectares composée de 6-7 lopins dispersés. Trois facteurs peuvent expliquer cette parcellisation : la distribution foncière en petites parcelles, réalisée à la suite des réformes agraires des années 1920, le morcellement des terres lors de l'héritage égalitaire et l'acquisition des propriétés foncières parcelle par parcelle en fonction des possibilités financières de chaque gospodaria.
}

loi, ces cinq descendants sont propriétaires de fait mais pas de jure parce que cette propriété appartient à la famille entière.

La restitution a donc forcé les héritiers à finaliser le processus de transmission, réalisé avec un retard de 45 ans et à partager la terre héritée, ce qui a créé une situation juridique extrêmement compliquée. Sur 25 familles interviewées, seulement une avait procédé de jure au partage de la propriété foncière, les procédures juridiques étant très coûteuses. Presque tous les héritiers, villageois ou citadins, sont des propriétaires en indivision des terres extravilan ${ }^{10}$ restituées. D'ailleurs, il y a quelques villageois qui envisagent de prendre en compte les marges de manœuvre laissées par les prescriptions du droit civil. Gabriel Bandea (56 ans) par exemple, est au courant de tous les artifices juridiques. Même s'il est héritier unique, il s'est déjà renseigné auprès des notaires sur la meilleure façon de contourner la loi :

«Je suis allé chez un notaire et c'est lui qui m'a montré comment faire pour contourner la loi. Pour sortir de l'indivision par exemple, il faut faire un contrat d'achat-vente intra-tabulaire entre les parties concernées : cela coûte moins cher qu'un partage juridique normal. »

Il est cependant à noter que pour accéder à ces artifices juridiques, il faut disposer d'argent et d'information. Aller chez un notaire, se renseigner, payer des frais, ce sont des choses inaccessibles pour la plupart des villageois.

\section{Usages des terres et du paysage rural Un retour aux anciennes pratiques}

Le village de Cerghid est demeuré jusqu'à l'arrivée du communisme, un village de petits propriétaires terriens qui organisaient

10. La situation est très différente pour les terres intravilan (lopins jardinés) d'une superficie qui varie de 7 à 40 ares et qui ont une situation juridique réglementée. 
l'essentiel de leur vie autour des exploitations agricoles : la terre agricole était repartie en très petites propriétés. ${ }^{11}$ Les registres agricoles et les cadastres des années 1950 offrent une image détaillée de la structure et des modes de tenure de la propriété foncière avant la collectivisation. Ces documents d'archives montrent qu'avant l'époque communiste, la propriété foncière du village était organisée selon un système traditionnel qui reposait sur la distinction entre les communaux (terres exploitées en commun) et le terroir (propriétés individuelles intravilan et extravilan). Ainsi, le village disposait de terres agricoles et non-agricoles destinées à la construction des maisons et des dépendances.

Les biens communaux étaient constitués de forêts et pâturages ( 375 hectares environ). Avant la collectivisation, ces biens ont été exploités et administrés par la communauté villageoise. L'ancien système de propriété accordait une place importante au terroir (terre agricole de propriété individuelle) situé également en extravilan. Le registre agricole de 1957 montre que le territoire agricole extravilan couvrait une surface de 1100 hectares. Ces terres formaient un seul grand espace désigné par le mot hotar par les paysans de Cerghid. Celui-ci était découpé en lanières sous la forme de propriétés individuelles. Les propriétés étaient voisines les unes des autres et leurs limites formaient des lignes droites ; on y reconnaît les éléments de l'organisation sociale du village. Il n'y avait pas de clôtures. La délimitation

11. La réforme agraire de 1921 a changé la structure de la propriété foncière à l'échelle du pays. Elle prévoyait l'expropriation de 6 millions d'hectares dont 4 millions ont été distribués à 1,4 million de paysans. Les propriétés de plus de 100 hectares qui représentaient encore $48 \%$ des terres agricoles avant la réforme n'en représentaient plus que $8 \%$ après. La part des propriétés de moins de 10 hectares dans le total des terres agricoles est passée de $39 \%$ à $79 \%$. Cette réforme a fait de la Roumanie un pays de petits propriétaires en consolidant une paysannerie moyenne et indépendante (Otiman, op. cit.). des propriétés était marquée par des pierres, des piquets ou des arbustes. Ces simulacres de clôtures étaient appelés miezuime, ce qui veut dire « le milieu de l'espace ». Ce système de voisinage a été repris exactement de la même manière après la chute du communisme. L'absence de clôtures pour la délimitation des propriétés peut être expliquée autant par le système traditionnel de voisinage que par un lien généalogique avec la propriété foncière :

"Les paysans n'ont jamais pensé qu'ils auraient besoin de clôtures pour délimiter leurs propriétés. Chacun savait très bien où était située sa parcelle. Personne ne pouvait s'emparer d'un bien qui n'était pas à lui. Tout était basé sur le respect de la propriété et sur la confiance mutuelle. » (Ioan Hora, 64 ans)

"Avant la collectivisation les gens respectaient tous une règle sainte : chacun respectait sa place dans le hotar. » (Raluca Anton, 70 ans)

Cet attachement à la terre " ancestrale » se trouve également exprimé dans les dénominations attribuées aux propriétés foncières. Si la toponymie de certaines parties du hotar se réfère à la disposition spatiale (Dos, Gruiet, Copacel) et à la qualité de la terre (Baltoci, Tausoare, Mocirle), bon nombre d'autres parties du hotar ont gardé les noms des anciens propriétaires latifundiaires (Costa lui Victor, Viile Ilenei, Ritu lui Bucur) ou les noms qui réfèrent au voisinage géographique (Valea Suplacului, Valea Idrifaii). Ces dénominations se transmettent d'une génération à l'autre sans aucune modification. Ainsi, une personne qui n'est pas familiarisée avec ce type de dénominations comprend mal la description que les paysans font de leurs propriétés puisque les références à telle ou telle partie du hotar sont assez nombreuses.

La même situation s'applique aux unités de mesure des surfaces agricoles. Les paysans continuent à se référer à l'ancien système de mesure utilisé à grande échelle en Transylvanie qui s'appelle iugar. Celuici provient du latin jugerum et correspond à 57 ares $\left(5775 \mathrm{~m}^{2}\right)$. Une autre unité de 
mesure spécifique, toujours en vigueur dans la pratique villageoise, s'appelle loc de-o feldera, soit une parcelle qui produit une quantité de céréales qui vaut une feldera (double décalitre). L'étendue de cette parcelle est évaluée à 7 ares. Les hectares et les ares sont aussi largement adoptés par les villageois. Ils mettent les deux systèmes de mesure (local : jugare et officie : are, hectares) dans une relation diachronique qui renvoie à deux systèmes de gestion économique différents. Lorsqu'ils se réfèrent à la propriété d'avant collectivisation, ils utilisent le mot iugar alors que pour décrire la situation actuelle ils utilisent ares et hectares. La logique formelle et coutumière y coexistent ${ }^{12}$, ce qui exprime l'attachement à des valeurs et systèmes de pensée traditionnels.

Cette propriété extravilan (communaux et terroir) est entièrement collectivisée en 1962. Les changements introduits par le travail mécanisé et de grande surface affectent la structure de cette propriété. Le village a perdu 266 ha de terres de labour et 229 ha de pâturages entre 1965 et $1989^{13}$. La coopérative de Cerghid a constamment cédé des terres aux entreprises d'État et aux associations intercoopératives créées dans les villages voisins. Certaines surfaces détériorées, à la suite d'un glissement de terrain, deviennent pâturages alors que d'autres terres arables, situées sur le versant sud, sont transformées en vignes et vergers.

Après la chute du communisme, la propriété communautaire se recompose selon les anciennes règles d'avant la collectivisation. Ainsi, la communauté récupère le

12. Il y a des différences régionales concernant les unités « traditionnelles » de mesures agricoles. En Valachie par exemple, une vieille unité de mesure était le pogon (terme qui provient du bulgare) qui correspondait à $5012 \mathrm{~m}^{2}$.

13. Les cahiers statistiques de 1965 indiquent 975 ha de terres arables et 379 ha de pâturages de prés de fauche. En 1989, la coopérative détenait seulement 709 ha de terres de labour et 150 ha de pâturages de prés de fauche. droit d'usage sur les pâturages qui redeviennent la propriété de la commune. Le terroir est restitué aux anciens propriétaires. La commission de redistribution foncière, formée de deux ingénieurs de la commune et de six villageois, a avancé assez lentement dans le processus de redistribution foncière. Premièrement, la commission a dû affronter un déficit de surface arable de plus de 200 ha par rapport à l'avant collectivisation. Deuxièmement, chaque parcelle devait être confrontée avec le cadastre et les positions topologiques, telles qu'indiquées dans le registre agricole, tout en respectant les voisinages. Le désir des gens de récupérer les terres sur les anciens emplacements et le manque de spécialistes ont compliqué le processus effectif de mesure et de redistribution qui a duré presque un an pour la plupart des propriétés. Jusqu'en 2000 les titres fonciers ont été accordés à $60 \%$ des gospodarii. C'est seulement en 2003 que ce pourcentage atteint $99 \%$.

Pendant la période post-socialiste, la structure de la propriété foncière et les modalités de la travailler ont été très différenciées. On a affaire à un système duel de structures productives, moitié paysanne, moitié de grande surface. Les nouveaux propriétaires ont pris, selon leurs disponibilités techniques et financières, de petites parcelles jusqu'à 50 ares sur lesquelles ils cultivent du maïs, du blé et des plantes techniques avec des outils agricoles rudimentaires. Sur ces superficies réduites, chacun développe une petite polyculture autocentrée, cherchant à assurer l'approvisionnement familial en produits de base. Le total de ces parcelles travaillées individuellement ne dépasse pas 100 hectares de terres labourables. La plupart des familles interviewées affirment qu'elles n'ont pas les moyens financiers pour cultiver seules les parcelles. Le plus grand problème c'est le manque d'outillages agricoles. La dotation en machines demeure très largement en dessous des besoins villageois et la traction animale reste en usage. 
La deuxième catégorie, la plus nombreuse, est constituée de terres données en fermage ou laissées en friche (600 ha environ). Plusieurs options agricoles ont été envisagées sur ces surfaces. Entre 1991 et 1994, ces terres ont été cultivées par une association agricole initiée par quelques anciens gestionnaires de la coopérative qui ont essayé de s'adapter au nouveau contexte social. Elle a utilisé « les restes » de l'héritage collectiviste pour assurer son fonctionnement : les étables, les installations agricoles de la Coopérative agricole de production (CAP) et les anciens tracteurs de la Station de mécanisation agricole (SMA) ${ }^{14}$.

Le manque d'expérience en matière de gestion, la méfiance des villageois, la corruption, l'inflation galopante et la dissolution de la $\mathrm{SMA}^{15}$ ont conduit l'association agricole à la faillite. Après 10 ans, ses membres fondateurs sont traduits en justice à cause de crédits jamais remboursés. Entre 1995 et 2004 , le village est « sauvé » par quelques entrepreneurs qui ont pris les terres à bail. $\mathrm{La}$ situation a empiré les années suivantes. En 2010, 450 ha de terres extravilan sont travaillées par 5 petits entrepreneurs et les villageois. Le reste de 250 hectares est laissé en friche. Un investisseur allemand a manifesté son intérêt pour cultiver ces terres afin de faire fonctionner une fabrique de biocombustible mais la réussite de ce nouveau projet demeure bien incertaine.

Il y a seulement 10 familles qui cultivent l'ensemble des terres restituées avec leurs propres moyens. Pour ces familles, l'activité

14. Entreprise qui assurait les travaux mécanisés des coopératives.

15. Une disposition gouvernementale de 1993 prévoyait la dissolution des SMA. Ceux qui ont travaillé dans le cadre de cette structure (tractoristes, techniciens) avaient la possibilité d'acheter à des prix dérisoires les outillages agricoles qui se trouvaient dans le patrimoine des SMA. Les villageois déplorent cette mesure législative qui, au lieu de développer l'initiative privée, a conduit à la disparition du parc des machines agricoles. Les outillages agricoles ont été vendus ou détruits à la suite de ce démantèlement. agricole n'est plus l'unique source de travail et de revenu. Elles entrent dans un système complexe qui, au sein de la gospodaria, associe les différentes générations, y compris les branches aujourd'hui urbaines de la famille. Une autre catégorie est formée par des salariés qui travaillent en ville et qui s'occupent de l'agriculture à la fin de leurs journées de travail. Ils ont les moyens financiers pour assumer les coûts des travaux agricoles mais les revenus qu'ils gagnent leur permettent seulement d'assurer les besoins primaires pour la gospodaria :

«Je ne veux plus donner les terres en fermage puisque les entrepreneurs ne respectent pas le contrat, ils nous donnent une quantité de céréales moindre que celle établie. Si je le cultive tout seul, je peux assurer les besoins néces saires pour la famille et un petit surplus qui me permet de payer une partie des travaux agricoles. Je vois qu'une règle s'est généralisée : on ne peut jamais récupérer l'argent investi dans la terre. Je ne sais plus pourquoi je continue de la travailler puisqu'elle ne m'apporte aucun revenu. En effet, je la travaille parce que je l'aime, je me sens attaché à ce bien qui est maintenant à moi. »(Ion Horea, 39 ans)

Ce témoignage résume en effet le drame que ce village vit depuis la chute du communisme, celui de ces petits propriétaires terriens décapitalisés, déchirés entre le désir de travailler la terre et l'impossibilité de la cultiver. Selon Petru Cozma (52 ans), paysan de fin de semaine, cette agriculture peu productive est le résultat de deux facteurs : le manque de ressources financières et l'application d'un savoir-faire paysan peu compétitif. Il croit qu'il est possible d'obtenir de bonnes productions sur des petites parcelles :

"Je n'ai aucune formation dans le domaine agricole mais j'ai commencé à me renseigner sur les cultures, les engrais chimiques, les espèces à cultiver. J'ai lu des livres et j'ai cherché sur Internet. Je suis le seul dans le village qui cultive une variété de maïs productif qui donne de très bonnes récoltes mais qui coûte 6 fois plus cher que les variétés ordinaires. La plupart des villageois n'ont vraiment pas les moyens financiers pour faire une agriculture productive. " 


\section{Les ambivalences du rapport à la terre}

Entre ces deux solutions (agriculture duelle et individuelle double active), on retrouve l'exemple de propriétaires qui habitent au village et qui travaillent les terres en faisant appel aux anciennes formes de faire-valoir de la propriété foncière : le travail en quotepart $^{16}$. Le cas de Vladimir Giurgiu (56 ans) combine ces deux situations. Il a hérité 9 hectares 30 ares de ses parents et 5 hectares d'une tante qui est restée sans héritiers directs (16 hectares au total). Vladimir Giurgiu a trouvé deux solutions pour cultiver ses terres: une partie est travaillée en quote-part et une autre partie est donnée en fermage.

Même si ce propriétaire dispose d'une grande surface de terre, il ne se considère pas riche :

«Je ne fais pas fortune avec ces terres. La rente
en produits que je reçois chaque année suffit seu-
lement pour les besoins de la famille. J'aimerais
vendre une partie de mes terres mais cela ne vaut
pas la peine maintenant, il n'a pas de valeur sur
le marché. Je me demande cependant si cette terre
va valoir quelque chose dans un avenir proche. La
terre a toujours eu une valeur en soi, c'est pour-
quoi j'espère que son prix va augmenter. Même à
un prix rentable, je ne vendrais qu'une partie de
mes terres. Je dois laisser une surface de 5 ha à
mon enfant pour qu'il hérite quelque chose de
son père et de son grand-père. Si la terre n'ac-
quiert pas une valeur marchande, au moins elle
aura une valeur sentimentale pour mon fils dans
le sens qu'il recevra quelque chose de ses aïeuls. »

16. Avant la collectivisation, la forme de faire-valoir la plus utilisée était le travail en quote-part où la rente était toujours fixée en nature (céréales, plantes techniques). Il était réglementé par des arrangements verbaux entre les teneurs, d'habitude les grands propriétaires, qui n'avaient pas la force de travail nécessaire pour cultiver la terre et les paysans pauvres pour lesquels ce type de travail était une modalité de survie. Le preneur assurait seulement la partie manuelle du travail agricole (binage, récolte, fauchage, etc.) alors que le propriétaire prenait les décisions concernant les types de cultures et assurait les moyens techniques pour la mettre en marche. Le travail accompli, le bailleur gardait $2 / 3$ de la récolte alors que le preneur avait le droit à $1 / 3$.
Ce témoignage, fait par un propriétaire qui déclare ne pas se sentir attaché à son bien foncier, peut surprendre à première vue. En même temps, il révèle l'ambivalence du rapport à la terre. D'un côté, en l'absence d'une valeur marchande et d'outils pour la cultiver, la terre est vue comme une corvée. Tous les changements que le village a vécus ont affecté le rôle de la terre dans l'organisation économique et dans la reproduction sociale. Elle ne constitue plus un élément fondamental, comme c'était le cas dans la société traditionnelle, puisque la plupart des villageois ont d'autres sources de revenu : une pension de retraite, un salaire, une aide de la part des enfants. En même temps, comme en font foi toutes les entrevues, les villageois et les citadins attachent à la terre une valeur en soi. Le manque de valeur marchande est perçu comme temporaire et déterminé par un contexte économique défavorable. Cette perception est soutenue par la quasi-absence d'un marché foncier : 20 transactions foncières ont été enregistrées en 20 ans. Les prix des terres extravilan restent toujours bas : 2000 le $i^{17} /$ hectare en moyenne, ce qui équivaut à 500 euros. Pour les terres intravilan, les prix sont beaucoup plus élevés : 2000 lei/aire ce qui équivaut à 50000 euros pour un hectare. Les différences sont énormes et le marché foncier rend bien compte de la dynamique d'intérêts économiques qui se déplacent de la terre en extravilan à la terre en intravilan. Ce déplacement signifie que la maison et la gospodaria commencent à être plus valorisées que le patrimoine foncier. Ces transactions foncières, même peu nombreuses, reprennent les mêmes pratiques coutumières d'avant la collectivisation. En règle générale, les actes d'achat-vente ne sont pas présentés et enregistrés devant le notaire :

"J'ai acheté 7 ares de terre il y a 2 ans. Nous avons signé un acte d'achat-vente qui n'est pas

17. Monnaie roumaine (sg. leu, pl. lei). Un euro $=$ 4 lei. 
enregistré. Tout le monde fait comme cela car les taxes notariales et de cadastre sont très élevées. Elles dépassent largement la valeur de la terre.» (Crin Popescu, 52 ans)

D'un autre côté, les pratiques des villageois démontrent que la terre revêt encore une grande valeur symbolique. Premièrement, elle a une valeur réparatrice, de bien récupéré qui reconvertit les anciens membres coopérateurs en propriétaires et qui légitime, après 45 ans de communisme, le droit à la propriété :

"Après que j'aie reçu la terre héritée de mon père, j'ai commencé à me sentir très attachée à elle. Pendant la collectivisation, c'était différent, cela ne m'intéressait pas, maintenant je la travaille avec beaucoup d'affection puisqu'elle m'appartient. » (Iulia Cernea, 62 ans)

Deuxièmement, la loi de la restitution ravive les liens généalogiques à la terre en partie brisés par le régime collectiviste, en rappelant à chacun, par la force des relations de filiation et d'alliance, que la terre est d'abord un bien qui lie les générations d'un groupe familial, les vivants et les morts. Une valeur patrimoniale qui l'emporte sur la valeur marchande. Rusu Valeriu, qui s'occupe des transactions foncières au village, affirme que seulement les villageois sans descendants sont intéressés à vendre la terre. Cette logique s'accorde parfaitement avec la situation exposée ci-dessus du propriétaire Vladimir Giurgiu qui veut laisser une partie de ses terres comme héritage à son fils. Laisser les héritiers sans terre est perçu comme une catastrophe car on met en péril la continuité de la lignée: "C'est une honte de vendre la terre. Seulement les Tsiganes vendent leurs terres ». Ce rapport ambivalent à la terre ne laisse pas de place à l'indifférence. Corvée, moyen de subsistance ou capital symbolique, la terre suscite toujours des discussions dans la communauté.

Comment comprendre la nature de tous ces changements et l'échec des solutions agricoles envisagées jusqu'à présent ? S'agit-il de l'expression d'un rejet de l'hé- ritage de l'expérience socialiste ou d'une volonté de retour à l'expérience pré-collectiviste animée par un fort attachement à la terre ? De ce point de vue, on retrouve une situation hybride qui combine plusieurs variables à la fois.

Les entretiens avec les villageois conduisent à nuancer les appréciations à l'égard de la nouvelle agriculture. Quand on leur demande quels contextes fonciers et agricoles leur ont été les plus favorables, 24 des 25 familles interviewées ont répondu sans hésitation être heureux de retrouver leur terre, invoquant chaque fois la liberté retrouvée et la possibilité de bénéficier des fruits de leur travail :

\begin{abstract}
«Pour nous, c'est mieux maintenant puisque ce que nous travaillons c'est à nous, il n'y a plus personne pour nous diriger et nous commander, on peut organiser nous-mêmes notre travail. » (Valeria Sita, 63 ans)
\end{abstract}

Mais quand on les interroge sur les difficultés de la mécanisation, les réponses se font plus nuancées. Tous les villageois, sans exception, répondent regretter les travaux mécanisés de l'organisation collective. Ils sont conscients que ce travail « primitif » de la terre ne peut pas durer et que l'agriculture doit changer de face :

« L'avenir est le travail mécanisé, sur des grandes surfaces. La solution la plus envisageable est l'affermage, il nous faut un entrepreneur sérieux. Ce travail primitif sur des petites parcelles ne peut plus durer. Il va perdurer peut-être jusqu'à la disparition de notre génération (60-70 ans) qui est encore habituée à travailler de cette manière. » (Tiberiu Voda, 67 ans)

L'avenir demeure incertain et pour certains, tout semble dépendre de la volonté divine :

«Les gens ont perdu l'espoir, ils sont épuisés après 45 ans de communisme et 20 ans de transition sauvage, ils n'ont plus ni les moyens ni les forces pour trouver des solutions. Seulement quelque chose de l'extérieur peut nous sauver, on attend toujours qu'un miracle divin se produise et qu'on puisse travailler les terres comme il faut. » (Dumitru Uciu, 44 ans) 
Les villageois critiquent également la logique distributive de la loi 18/1991. Ils sont mécontents que la loi ait donné la possibilité aux ingénieurs et aux techniciens de devenir propriétaires. Au dire des villageois, cette nomenclatura de l'agriculture socialiste a même profité de cette disposition de mise en propriété et a pris les terres de meilleure qualité. Les paysans montrent du doigt la corruption des commissions foncières, le coût élevé des travaux agricoles, le manque de subventions étatiques, les bas prix des produits agricoles, bref la classe politique. Les acteurs ruraux ne se perçoivent pas comme agents de transformation, le salut doit venir de l'extérieur de la communauté. Ils se sentent impuissants face à la structure et c'est dans ce sens qu'on les perçoit comme «passifs » (Verdery, 2002a). Comme dans le cas de la coopérative, ils ne peuvent pas changer la structure mais seulement développer des petites stratégies et en tirer profit pour survivre. Les vrais enjeux sont laissés aux « grands » : politiciens, anciens gestionnaires de la coopérative, entrepreneurs. La stratification sociale se fait sur un background politique.

\section{Conclusion}

Les résultats de cette recherche permettent de nuancer certains postulats simplificateurs sous-tendus par une bonne partie de la littérature scientifique consacrée aux changements post-socialistes qui véhicule l'idée d'une transition unilinéaire et prédictible du socialisme au capitalisme. Les comportements analysés dans le village de Cerghid relèvent d'une transition contradictoire, fluide et pluridimensionnelle qui englobe passé et présent, traditionnel et modernité et qui amène une diversification sans précédent des pratiques familiales et agricoles.

Cette recherche montre également que la résurgence des pratiques coutumières de transmission patrimoniale a directement influencé le processus de décollectivisa- tion en Roumanie. Le retour à la pratique coutumière d'indivision foncière entraîne des stratégies diverses de partage qui vont du renforcement des solidarités familiales à de nombreux conflits et tensions. La tension entre la légitimité du droit civil et la légitimité des règles coutumières est la source principale des conflits entre les parents collatéraux.

Les représentations de la propriété foncière renvoient à deux logiques contradictoires : une logique/rapport marchand (économique) et une logique/rapport affectif (symbolique). Dans le contexte des relations économiques de production, la terre commence à acquérir une conception laïcisée et marchande. Plusieurs villageois sont prêts à donner les terres en fermage ou à trouver d'autres solutions pour que cellesci ne soient pas laissées en friche. Dans celui des relations familiales, la terre représente une valeur symbolique et un lieu de mémoire. Elle joue un rôle moins important dans l'organisation de la reproduction sociale. La gospodaria devient l'élément matériel de base qui assure la continuité et le devenir familial.

En Roumanie, le paysage de la décollectivisation varie sensiblement d'une région à l'autre. Les solutions agricoles propres à chaque communauté paysanne sont le résultat d'une gamme de facteurs (pratiques économiques et juridiques, héritage collectiviste, rapport à la terre, qualité de la terre), ce qui rend difficile la construction d'un modèle unitaire de ce processus à l'échelle du pays. À première vue, le village de Cerghid, avec un bon potentiel agricole, situé à 15 kilomètres de deux centres urbains, aurait dû être un modèle de réussite en matière de développement agricole après la chute du communisme. Or, les résultats de cette étude révèlent une réalité tout autre. La communauté de Cerghid s'est trouvée démunie devant une transition qui n'offrait pas un modèle de référence de sortie du collectivisme. Ce manque de mobilisation économique est doublé par 
les luttes entre les héritiers pour partager la terre et la situation non réglementée des propriétés foncières restituées qui se trouvent en indivision juridique. Cette situation favorise le déploiement d'un ensemble d'artifices procéduriers afin de contourner ou d'exploiter les lacunes et les ambiguïtés de la législation en vigueur.

L'adhésion de la Roumanie à l'Union européenne en 2007 n'a pas réussi à changer la dynamique du développement dans les campagnes roumaines. Les autorités politiques et les populations rurales sont encore peu préparées à intégrer les programmes de développement sans précédent mis en place (Hirschhausen, 2008). Les changements risquent d'être longs et pleins de conséquences imprévues et paradoxales.

\section{RÉFÉRENCES BIBLIOGRAPHIQUES}

Amblard L., Colin J.-P. (2006). Dimensions organisationnelles et pratiques contractuelles foncières : les sociétés agricoles en Roumanie. Paris, SFER, Économie rurale, $\mathrm{n}^{\circ} 293$, p. 55-71.

Badescu I. (dir.) (2003). Taranii si noua Europa. Bucuresti, Amacord.

Cartwright A.-L. (2001). The Return of the Peasant. Land Reform in Post-Communist Romania. Dartmouth, Asghate.

Civici A., Jouve A.-M. (2009). Enchâssement social et politique de la propriété foncière dans les Balkans. Options Méditérranéennes, A82, p. 5-19.

Derouet B., Goy J. (1998). Transmettre la terre. Les inflexions d'une problématique de la différence. In Bouchard G., Goy J., Head-König A.-L. (dirs.), «Problèmes de la transmission des exploitations agricoles » (XVIII -XXe siècles). Rome, École française de Rome. p. 117-153.

Gaborean F. (2008). Reproduction familiale et changement social en milieu rural. Décollectivisation et pratiques successorales en Roumanie. Québec, Université Laval, Anthropologie et sociétés, 32 (1-2), p. 65-83.

Gambold L.-L., (2008). L'antagonisme de l'exploitation collective postsocialiste. Québec, Université Laval, Anthropologie et sociétés, 32 (1-2), p. 23-42.

Frybes M. (dir.) (1998). Une nouvelle Europe centrale. Paris, La Découverte.
Hann C. (dir.) (2002). Postsocialism. Ideals, Ideologies and Practices in Eurasia. London \& New-York, Routledge.

Hirschhausen (Von) B. (1997). Les nouvelles campagnes roumaines. Paradoxe d'un retour paysan. Paris, Belin.

Hirschhausen (Von) B. (1999). Boycotter l'histoire ? Le défi de la modernité pour les campagnes roumaines. La nouvelle Alternative, $\mathrm{n}^{\circ} 3$.

Hirschhausen (Von) B. (2008). Les sociétés roumaines face à l'irruption des programmes de développement. Revue d'études comparatives Est-Ouest, vol. 39, $\mathrm{n}^{\circ} 4,230 \mathrm{p}$.

Le Meur P.-Y. (2002). Approche qualitative de la question foncière. Note méthodologique. Document de travail de l'unité de recherche 095, IRD REFO, 8 p.

Lhomel E. (1995). La décollectivisation des campagnes roumaines : incertitudes et enjeux. Revue d'études comparatives EstOuest, vol. 26.

Maurel M.-C. (dir.) (1994). La transition post-collectiviste. Paris, Harmattan.

Mihailescu V. (2000). Masnie diffuse, du communisme au capitalisme : questions et hypothèses. Balkanologie, vol. 4, $\mathrm{n}^{\circ} 2$.

Mihailescu V. (1996) Doua sate in tranzitie. Tipuri strategice dominante in lumea rurala. Revista de Cercetari Sociale, $\mathrm{n}^{\circ} 3$. Otiman P.-I. (1994). Agricultura Romaniei la cumpana dintre mileniile II si III. Timisoara, Editura Helicon. 
Sandu D. (1999). Cine sunt antreprenorii din agriculture de tranzitie. Sociologie Romaneasca, $\mathrm{n}^{\circ} 1$.

Stan S. (2005). L'agriculture roumaine en mutation. La construction sociale $d u$ marché. Paris, CNRS Éditions.

Turner B., Wiber M.-G. (2009). Conjonctions paradoxales : propriété rurale et accès aux ressources dans un environnement transnational. Ottawa, Wilfrid Laurier University Press, Anthropologica, vol. 51, $\mathrm{n}^{\circ} 1$, p. 15-28.
Verdery K. (2003). The Vanishing hectare: Property and Value in Postsocialist Transylvania. Ithaca/New-York/London, Cornell University.

Verdery K. (2002a). Seeing like a major: or How Local Officials Obstructed Romanian Land Restitution. Ethnography, vol. $3, \mathrm{n}^{\circ} 1$.

Verdery K. (2002b). Property and Time in Transylvania's Land Restitution. Anthropological Theory, vol. $1, \mathrm{n}^{\circ} 3$.

\section{LOIS ET DOCUMENTS JURIDIQUES}

La loi 18/1991, publiée le 19 février 1991, La loi 1/2000, publiée le 10 janvier 2000, 123 articles. 41 articles.

La loi 169/1997 qui modifie et complète la Le code civil roumain de 1865, modifié et loi 18/1991, publiée le 4 novembre 1997, complété, édition de 1993.

7 articles. 